\section{Lords on Europe's biotechnology}

SIR-You have published (Nature 327, $650 ; 1987)$ an account of the UK House of Lords' report, Biotechnology in the Community, under the headline "European biotechnology plans leave the Lords cold". I find your report disappointing in that it neglects the quite positive support for many of the ideas expressed in the Commission discussion paper, COM(86)221, concerning agro-industrial development. Thus their lordships advocate "the extension of Community action in biotechnology and agro-industrial development into some of the suggested pilot projects".

This positive statement is accompanied by many helpful suggestions on the programme content, by a statement that the maximum funding of individual projects should be increased above the "very low level" available within earlier biotechnology programmes and by the advice (mentioned by your correspondent) that the Community should concentrate on projects not funded elsewhere and that are likely to show promise of economic viability.

The programme proposal we are now preparing will take into account their lordships' helpful comments, as well as the many other comments we have received and the 856 responses from European industry, research institutes and academic institutions that followed our call for expressions of interest in such a programme made last year.

The initial programme will be modest, in line with the reduced resources of the overall Framework Programme; but we are convinced that through launching support for a few worthwhile projects, we can promote increased land use and novel industrial development, utilizing the recent developments in biotechnology, and aiding the continuing evolution towards a market-driven agriculture.

Directorate-General for Science,

P. Fasella

Research and Development,

Joint Research Centre,

Commission of the European Communities, B-1049 Brussels, Belgium

\section{Germ of an idea}

SIR-We have twice ${ }^{1.2}$ been subjected to undocumented speculation by Hoyle and Wickramasinghe, who hypothesize that novel influenza virus variants come to us through seeding from comets. They state that the "epidemiological evidence is consistent with a model where the causative agent...is airborne and is brought down seasonally from a reservoir established in the stratosphere".
Nature not require Hoyle and Wickramasinghe to provide experimental evidence for their views? Where are the data showing that fragile lipid-containing influenza viruses survive extraterrestrial travel and when have influenza viruses been isolated from the stratosphere?

The data are equally consistent with simpler, more plausible explanations for which supporting data exist. For example, there is overwhelming sequencing evidence that influenza viruses undergo sequential evolutionary changes in nature with clear-cut ancestor-progeny relationships $^{3}$. Furthermore, the appearance of novel influenza virus subtypes in man is clearly explained by a simple genetic exchange (reassortment of genes) of human and animal influenza viruses ${ }^{4}$, thus eliminating the need for seeding by comets. It appears untenable to ignore scientific evidence in favour of unsupported outer-space (spaced-out?) theories.

Department of Microbiology,

Peter Palese

Mount Sinai School of Medicine,

1 Gustave L. Levy Place,

New York, New York 10029, USA

ROBERT WEBSTER

St Jude Children's Research Hospital,

332 N. Lauderdale, POB 318,

Memphis, Tennessee 38101, USA

1. Hoyle, F. \& Wickramasinghe, N.C. Nature 322, 509-511 (1986)

Hoyle, F. \& Wickramasinghe, N.C. Nature 327, 664 (1987)

3. Buonagurio, D.A. et al. Science 232, $980-982$ (1986)

4. Webster, R.G. et al. Nature 296, 115-121 (1982).

\section{But whose genome?}

SiR-Word has reached those of us who work in the hinterlands of biology (on plants) that a project is afoot to sequence the human genome. Of course this raises ethical and practical questions. The one I like best is, "Whose genome is going to get sequenced?" May I make some suggestions? To begin with there is the problem of race, which is at least partly solved as it seems that at least one Asiatic and one Caucasian project are already in the works. The next question is that of nationality. It seems that every country that can afford it is going to have to have its own project. This line of reasoning continues until we get to the choice of the individual ( $R$. Reagan, M. Thatcher, F. Mitterrand ... .) and then there are the social problems: class, accent and so on. As I see it, the human genome sequencing project will never get off the ground until a compromise can be found. My suggestion is that it go out to tender. This might help with the funding. Unfortunately, J.P. Getty and $H$. Hughes are dead, but there must be somebody who can afford to be sequenced.

DAVID TEPFER

Laboratoire de Biologie de la Rhizosphere, Institut de la Recherche Agronimique, 78000 Versailles, France

\section{Eiffel Tower in space}

SIR-The protests against the still-evolving concept of an 'Eiffel Tower in space' (Nature 326, 125; 1987) are reminiscent of similar protests mounted against the original Eiffel Tower, the Statue of Liberty and, indeed, almost every monumental edifice that has risen above the horizontal. Recent complaints from the astronomical community may be more serious, but there are several mitigating factors.

(1) The proposed structure would be a circle $\leqslant 30$ arc-minutes in diameter of starlike points of light resembling Vega in brightness and having an integrated visual magnitude $\geqslant-5$. It would be in the local sky at any given point on the Earth for only a few minutes of every night. Although the light from the sculpture might affect photometric astronomical observations in the rather improbable circumstance that it should visit the vicinity of the small regions of sky being observed, the general effect would be slightly greater than that of the planet Venus and much less than that of the Moon. As the orbit will be known, astronomers can easily plan their observations to avoid the problem.

(2) Although astronomical photographs showing 'contamination' by faint satellites are well known, these are usually wideangle deep-survey pictures. There will be only one 'Eiffel Tower' structure, along with Soviet and US space stations and a few other man-made objects brighter than 1st magnitude.

(3) There is a lot of space, and the probability of collisions with useful satellites can be made ignorably small by judicious choice of orbit.

(4) The future of astronomy will be built in space, not on the Earth. Easy and inexpensive access to space, as provided by reusable aerospace planes and heavy-lift launch vehicles, will transform astronomy. The enormous scientific payoffs from the relatively modest space astronomy payloads launched so far amply demonstrate the importance of maintaining public interest in space development - interest that the 'Eiffel Tower' will certainly enhance.

Just as the original Eiffel Tower was a celebration of the progress and promise of engineering, so the new Eiffel Tower in space will be a celebration of the promise of space for the twenty-first century. I hope that this daring concept succeeds. I hope the critics will reflect on the beneficial interpretations and turn their attention instead to the real threats to the night sky such as air pollution and light pollution. The world can well use a new symbol of idealism and inspiration.

Washington, DC, USA
JOHN D. G. RATHER 